International Journal of Wireless \& Mobile Networks (IJWMN) Vol. 4, No. 2, April 2012

\title{
Mobile Agent BASEd Congestion CONTROL Using AODV Routing Protocol TECHNIQUE FOR MOBILE AD-HOC NETWORK
}

\author{
Vishnu Kumar Sharma ${ }^{1}$ and Dr. Sarita Singh Bhadauria ${ }^{2}$ \\ ${ }^{1}$ Department of CSE, JUET, Guna, Madhya Pradesh, India \\ vishnusharma97@gmail.com \\ ${ }^{2}$ Department of Elex, MITS Gwalior, Madhya Pradesh, India \\ Saritamits.61@yahoo.co.in
}

\begin{abstract}
In Mobile Ad hoc Networks (MANETs) obstruction occurs due to the packet loss and it can be successfully reduced by involving congestion control scheme which includes routing algorithm and a flow control at the network layer. In this paper, we propose to agent based congestion control technique for MANETs. In our technique, the information about network congestion is collected and distributed by mobile agents (MA) A mobile agent based congestion control AODV routing protocol is proposed to avoid congestion in ad hoc network. Some mobile agents are collected in ad-hoc network, which carry routing information and nodes congestion status. When mobile agent movements through the network, it can select a less-loaded neighbor node as its next hop and update the routing table according to the node's congestion status. With the support of mobile agents, the nodes can get the dynamic network topology in time. By simulation results, we have shown that our proposed technique attains high delivery ratio and throughput with reduced delay when compared with the different existing technique.
\end{abstract}

\section{KEYWORDS}

AODV routing protocol, Congestion control Mobile Ad hoc Networks (MANETs), Mobile Agents (MA), Total Congestion Metric (TCM), Enhanced Distributed Channel Access (EDCA), Transmission opportunity limit (TXOP).

\section{INTRODUCTION}

The mobile ad-hoc network is accomplished of forming a temporary network, without the require of a central administration or standard support devices available in a conventional network, thus forming an infrastructure-less network. In order to guarantee for the future, the mobile ad hoc networks establishes the networks everywhere. To avoid being an perfect candidate during rescue and emergency operations, these networks do not depend on the irrelevant hardware. These networks build, operate and maintain with the help of constituent wireless nodes. Since these nodes have only a limited transmission range, it depends on its neighboring nodes to forward packets [1].

Obstruction control is a key problem in mobile ad-hoc networks. The standard TCP congestion control mechanism is not able to handle the unique properties of a shared wireless multihop channel well. In particular the frequent changes of the network topology and the shared nature of the wireless channel pose significant challenges.

Many approaches have been projected to overcome these difficulties [3] Ad-hoc network is a wireless and with no fixed apparatus (such as base stations) distributed network which is component of mobile terminals [27], each mobile terminal is not only host computer but also router.

DOI : 10.5121/ijwmn.2012.4220 
International Journal of Wireless \& Mobile Networks (IJWMN) Vol. 4, No. 2, April 2012

As power and bandwidth restrictions, ad-hoc network routing protocols should allocate routing tasks literally in the mobile nodes. At present, AODV routing protocol is often used in ad-hoc network. But its biggest failing is delay. In routing discovery and maintenance, a large number of data is transmitted through a small number of nodes is hop to lead to network congestion and bottleneck. At the same time, unwarranted data load will be exhaust nodes energy rapidly. With the increase of brownout nodes, network connectivity will be weakened and network overall survival time will be shorten subsequently. Therefore, In order to balance the network load and maintain network continuous, efficient and stable operation, it is necessary to take into account the routing nodes load and congestion in network [28]. In mobile ad hoc wireless network, mobile agent has mobility and autonomy. Therefore, it can be used to solve the ad hoc network congestion [26].

Ad-hoc networks are characterized by a need of infrastructure, and by a random and quickly varying network topology; thus the need for a robust dynamic routing protocol that can accommodate such an environment. Therefore, many routing algorithms have come into existence to satisfy the needs of communications in such networks. Recital comparison between two routing algorithms, AODV, from the immediate family and DSDV, from the proactive family. Both protocols were simulated using the ns-2 and were compared in terms of average throughput, packet loss ratio, and routing overhead, while changeable number of nodes, speed and pause time. Simulation exposed that although DSDV completely scales to small networks with low node speeds, AODV is favored due to its more efficient use of bandwidth [31].

\subsection{Congestion Control in MANETs}

Congestion takes place in MANETs with limited resources. In these networks, shared wireless channel and dynamic topology leads to interference and fading during packet transmission. Packet victims and bandwidth dilapidation are caused due to congestion, and thus, time and energy is wasted during its recovery. Congestion can be prevented using congestion-aware protocol through bypassing the affected links [2]. Severe throughput degradation and massive fairness problems are some of the identified congestion related problems. These problems are generated from MAC, and protocol routing and transport layers [3].

Congestion control is the main problem in ad-hoc networks. Congestion control is associated to controlling traffic incoming into a telecommunication network. To avoid congestive crumple or link capabilities of the intermediate nodes and networks and to reduce the rate of sending packets congestion control is used extensively [4]. Congestion control and dependability mechanisms are combined by TCP to perform the congestion control without explicit feedback about the congestion position and without the intermediate nodes being directly intermittent [4]. Their principles include packet conservation, additive increase and multiplicative decrease in sending rate, stable network. End system flow control, network congestion control, network based congestion avoidance, and resource allotment includes the basic techniques for congestion control [5].

Packet failure in MANETs is primarily caused due to obstruction. The packet loss can be condensed by involving congestion control over a mobility and failure adaptive routing protocol at the network layer. The congestion non-adaptive routing protocols, leads to the following difficulties:

- Long delay: The congestion control mechanisms takes much time for detecting congestion. Usage of new routes in some critical situations is advisable. In an ondemand routing protocol, the main problem is the delay stirring for route searching. 
International Journal of Wireless \& Mobile Networks (IJWMN) Vol. 4, No. 2, April 2012

- High overhead: It takes effort in new routes for processing and communication for discovering it. It also takes effort in multipath routing for maintaining the multi-paths, though there is another protocol.

- Many packet losses: The packets may be lost when the congestion is detected. To decrease the traffic load, a congestion control solution is applied either by decreasing the sending rate at the sender, or dropping packets at the intermediate nodes or by both methods. But high packet loss rate or a small throughput occurs at the receiver [6].

\subsection{Problem discovery and Proposed Protocol Overview}

Congestion adaptive routing has been examined in several studies. Estimating or reviewing the level of activity in the intermediate nodes using load or delay measurement, is the common approach in all the studies mentioned. The favorable path is established based upon the collected information, which helps in avoiding the existing and developing congested nodes. The performance of routing protocols is affected by the service type of the traffic carried by the intermediate nodes. But no research has stated this so far.

Before presenting themselves as aspirant to route traffic to the destination, the MANETs do not take the status of the queues into account, for the route discovery process. Because of this, the newly arriving traffic face long delays, packet drops, and fail to be transmit ahead of the already queuing traffic.

The mobile ad hoc networks performances are subjective to the congestion problem. A routing algorithm and a flow control scheme, includes the congestion control scheme. Enhanced performance and better congestion control can be achieved only by considering the routing and the flow control together. This was not done in earlier researches [12].

AODV routing protocol [30] is a distance vector routing protocol based on demand. The main characteristic is using a serial number to identify the routing is new or old and avoid routing loop. AODV routing protocol has the advantages of each intermediate node saves a routing request and response result implicitly to adapt to dynamic link rapidly .AODV protocol has two main components: routing discovery and routing maintenance.

\section{Related Work}

Yao-Nan Lien et al [7] proposed a new TCP congestion control mechanism by router-assisted approach. Their proposed TCP protocol, called TCP Muzha uses the assistance provided by routers to achieve better congestion control. To use TCP Muzha, routers are required to provide some information allowing the sender to estimate more accurately the remaining capacity over the bottleneck node with respect to the path from the sender to the receiver. With this information, TCP Muzha will be able to enhance the performance of both TCP and network.

Wei Sun et al [8] have compared the general AIMD-based congestion control mechanism (GAIMD) with Equation-based congestion control mechanism (TFRC TCP-Friendly Rate Control) over a wide range of MANET scenario, in terms of throughput fairness and smoothness. Their results have shown that TFRC and GAIMD are able to maintain throughput smoothness in MANET, but at the same time, they require only a less throughput than the competing TCP flows. Also their results show that TFRC changes its sending rate more smoothly than GAIMD does, but it gets the least throughput compares with TCP and GAIMD.

Yung Yi et al [9] have developed a fair hop-by-hop congestion control algorithm with the MAC constraint being imposed in the form of a channel access time constraint, using an optimizationbased framework. In the absence of delay, they have shown that their algorithm is globally stable using a Lyapunov-function-based approach. Next, in the presence of delay, they have shown that the hop-by-hop control algorithm has the property of spatial spreading. Also they 
International Journal of Wireless \& Mobile Networks (IJWMN) Vol. 4, No. 2, April 2012

have derived bounds on the "peak load" at a node, both with hop-by-hop control, as well as with end-to-end control, show that significant gains are to be had with the hop-by-hop scheme, and validate the analytical results with simulation.

Umut Akyol et al [10] have studied the problem of jointly performing scheduling and congestion control in mobile adhoc networks so that network queues remain bounded and the resulting flow rates satisfy an associated network utility maximization problem. They have defined a specific network utility maximization problem which is appropriate for mobile adhoc networks. They have described a wireless Greedy Primal Dual (wGPD) algorithm for combined congestion control and scheduling that aims to solve this problem. They have shown how the wGPD algorithm and its associated signaling can be implemented in practice with minimal disruption to existing wireless protocols.

S.Karunakaran et al [11] have presented a Cluster Based Congestion Control (CBCC) protocol that consists of scalable and distributed cluster-based mechanisms for supporting congestion control in mobile ad hoc networks. The distinctive feature of their approach is that it is based on the self-organization of the network into clusters. The clusters autonomously and proactively monitor congestion within its localized scope.

S.Venkatasubramanian et al [19] proposed the QoS architecture for Bandwidth Management and Rate Control in MANETs. The bandwidth information in the architecture can be used for QoS capable routing protocols to provide support to admission control. The traffic is balanced and the network capacity is improved as the weight value assists the routing protocol to evade routing traffic through congested area. The source nodes then perform call admission control for different priority of flows based on the bandwidth information provided by the QoS routing. In addition to this, a rate control mechanism is used to regulate best-effort traffic, whenever network congestion is detected. In this mechanism, the packet generation rate of the low-priority traffic is adjusted to incorporate the high-priority traffic.

R.Mynuddin Sulthani et al [20] proposed a joint design of reliable QoS architecture for mobile adhoc networks. In the reliable multipath routing protocol, dispersion and erasure code techniques are utilized for producing replicated fragments for each packet, to enhance reliability. Then messages with good delivery probability are identified and transmitted through the paths with high average node delivery index. While it receives an assured number of fragments, destination can recover the original packet. Next, a call admission control (CAC) scheme has been developed, in which, the calls are admitted based on the bandwidth availability of the path. Once congestion occurs, the best effort traffic is rate controlled, to free bandwidth for the real-time flows.

Lijun Chen et al [21] proposed the joint design of congestion control, routing and scheduling for ad hoc wireless networks. They formulate resource allocation in the network with fixed wireless channels or single-rate wireless devices as a utility maximization problem with schedulability and rate constraints arising from contention for the wireless channel. We also extend the dual algorithm to handle the network with time-varying channel and adaptive multi-rate devices, and surprisingly show that, despite stochastic channel variation, it solves an ideal reference system problem which has the best feasible rate region at link layer. In future, they will extend the results to networks with more general interference models and/or node mobility and further will enhance the performance gain from cross-layer design involving link layer.

Xuyang Wang et al [22] proposed a cross layer hop by hop congestion control scheme to improve TCP performance in multihop wireless networks which coordinates the congestion response across the transport, network, and transport layer protocols. The proposed scheme attempts to determine the actual cause of a packet loss and then coordinates the appropriate congestion control response among the MAC network, and transport protocols. The congestion 
International Journal of Wireless \& Mobile Networks (IJWMN) Vol. 4, No. 2, April 2012

control efforts are invoke at all intermediate and source node along the upstream paths directed from the wireless link experiencing the congestion induced packet drop.

Kazuya Nishimura et al [23] proposed a routing protocol that reduces network congestion for MANET using multi-agents. They use two kinds of agents: Routing Agents to collect information about congestion and to update the routing table at each node, and Message Agents to move using this information. In the future, they will investigate a better evaluation function and discuss the limits of its effectiveness. The evaluation function itself may change depending on the environment. Incorporating learning into the function is also an interesting issue.

Bhadauria,Sharma [24] proposed the information about network congestion is collected and distributed by mobile agents (MA). The MA measures the queue length of the various traffic classes and the channel contention and estimates the total congestion metric to find the minimum congestion level in the network. The congestion metric is applied in the routing protocol to select the minimum congested route.

P.K. Suri et al [29] proposed a bandwidth-efficient power aware routing protocol "QEPAR". The routing protocol is presented to minimize the bandwidth consumption as well as delay. QEPAR will help in increasing the throughput by decreasing the packet loss due to non availability of node having enough battery power to retransmit the data packet to next node. The proposed protocol is also helpful in finding out an optimal path without any loop

Vinay Rishiwal et al [30] proposed QoS based power aware routing protocol (Q-PAR). The selected route is energy stable and satisfies the bandwidth constraint of the application. The protocol Q-PAR is divided in to two phases. In the first route discovery phase, the bandwidth and energy constraints are built in into the DSR route discovery mechanism. In the event of an impending link failure, the second phase, a repair mechanism is invoked to search for an energy stable alternate path locally. Moreover the local repair mechanism was able to find an alternate path in most of the cases enhanced the network lifetime and delayed the repair and reconstruction of the route.

\section{Agent Based Congestion Control Routing}

\subsection{EDCA Mechanism of 802.11e}

The PCF and DCF modes have been replaced with HCF controlled channel access (HCCA), and enhanced distributed channel access (EDCA) which provides distributed access supplying service differentiation [13].

An extended version of the legacy DCF mechanism is EDCA. Access Categories (AC) or traffic priority classes like voice, video, best effort and background are defined by EDCA [14]. The access categories prioritize themselves from AC3 to AC0. In general, best effort and background traffic are maintained by $\mathrm{AC} 1$ and $\mathrm{AC} 0$ and real-time applications like voice or video transmission are maintained by $\mathrm{AC} 2$ and $\mathrm{AC} 3$ [15]. For the purpose of service differentiation, many MAC constraints vary with priority level chosen for each AC.

For the implementation of the EDCA contention algorithm the four transmission queues are applied with each AC being communicated with the others. The minimum idle delay before contention (AIFS), the Contention Windows (CWmin and CWmax), and the Transmission opportunity limit (TXOP) are the various parameters described here. The default values of each parameter are listed in Table 1. 
International Journal of Wireless \& Mobile Networks (IJWMN) Vol. 4, No. 2, April 2012

Table 1. IEEE 802.11e EDCA MAC System Parameters

\begin{tabular}{cccccc}
\hline $\begin{array}{c}\text { Access } \\
\text { Category }\end{array}$ & AIFSN & CWmin & CWmax & $\begin{array}{c}\text { Queue } \\
\text { length }\end{array}$ & $\begin{array}{c}\text { Max. } \\
\text { retry limit }\end{array}$ \\
AC3 & 2 & 7 & 15 & 25 & 8 \\
AC2 & 2 & 15 & 31 & 25 & 8 \\
AC1 & 3 & 31 & 1023 & 25 & 4 \\
AC0 & 7 & 31 & 1023 & 25 & 4 \\
\hline
\end{tabular}

In the MAC layer, voice traffic is conveyed through AC3 and the video traffic is conveyed through AC2 in accordance with 802.11e EDCA standard. The AC class differentiation in EDCA is very much useful in providing services to the traffic. Superior servicing is done for high-priority traffic and not much importance is given for low-priority traffic. The contention parameters of EDCA are not able to adapt to the network conditions, in spite of the delay sensitivity of real-time traffic taken into account. This leads to limitations in the QOS improvement [16].

ACs pause for diverse values of Arbitration Interframe Space (AIFS) and AIFSi is computed by,

$$
\text { AIFSi }=\text { SIFS }+ \text { AIFSNi } \times \text { SlotTime }
$$

where AIFSi is a positive integer which is greater than one, AIFSNi is the AC-specific AIFS number; SIFS and Slot Time are dependent on physical layer [14]. If the values of the subsequent parameters are small, the channel access delay will become less for the AC which leads the higher priority to approach the medium.

When a particular QoS station (QSTA) has the concession to begin transmissions, then the TXOP is expressed as the time interval in IEEE 802.11e. The initiation of the TXOP and the multiple frame transmission within an EDCA TXOP are the nodes approved by TXOP. The former occurs only when the EDCA rules allow entry to the medium. And the later occurs when an EDCA Function (EDCAF) holds the concession to contact the medium after completing a frame exchange sequence. The period of TXOP values are herewith in the EDCA parameter engraved in beacon frames. A STA is allowed to transmit multiple MAC protocol data units (MPDUs) from the same AC with a SIFS time interval between an ACK and the succeeding frame transmission. A single MPDU may be forwarded for each TXOP if the TXOP limits the value of 0 [17].

\subsection{Mobile Agent (MA)}

Each node has a routing table that stores $\mathrm{k}$ fresh routing information records from itself to every node $S:[S,\{(T c i, N H i, A N i, N P i) \cdots(T c m, N H m, A N m, N P m)\}]$, where $T c 1>T c 2>\cdots>T m$. We call $\mathrm{m}$ the number of entries. For each $i(1 \leq i \leq m), T c i$ is a time of visiting the adjacent node $A N i, N H i$ is the number of hops and $N P i$ is the number of MAs on ANi. When MA with the history $(S, T c, N H, A N, N P)$ visits a node $N$, the routing information on that node

$[S,\{(T c i, N H i, A N i, N P i) \cdots(T c m, N H m, A N m, N P m)\}]$ is updated to

$[S ;\{(T c, N H ; A N, N P),(T c i, N H i, A N i, N P i, N P i) \cdots$

(Tcm-1, NHm-1, ANm-1,NPm-1)\}] 


\subsection{Queue Length Estimation}

Our goal is to acquire macroscopic network statistics using a heuristic approach. We compute the traffic rate as follows: Let the value $L_{O}$ represent the offered load at the queue of node $i$ and it is defined as

$$
L_{o i}=\frac{A R_{i}}{S R_{i}}
$$

where $A R_{i}$ is the aggregate arrival rate of the packets produced and forwarded at node $i$ while $S R_{i}$ is the service rate at node $i$, i.e., $S R_{i}=1 / T$ where $T$ is the computed exponentially weighted moving average of the packets' waiting time at the head of the service queue. The distribution of the queue length $P R\left(Q_{1}\right)$ (essentially this is the probability that there are $Q_{l}$ packets in the queue) at the node is computed as

$$
P R\left(Q_{1}\right)=\left(1-L_{o i}\right) L_{o i}^{1}
$$

For $N$ distinct queues, the joint distribution is the product

$$
P R\left(Q_{11}, Q_{12} \cdots Q_{1 N}\right)=\prod_{i=1}^{N}\left(1-L_{o i}\right) L_{o i}^{l i}
$$

\subsection{Channel Contention Estimation}

IEEE 802.11 MAC with the distributed coordination function (DCF). It has the packet sequence as request-to-send (RTS), clear-to-send (CTS), data and acknowledgement (ACK). The amount of time between the receipt of one packet and the transmission of the next is called a short inter frame space (SIFS). Then the channel occupation due to MAC contention will be

$$
C_{\text {OCC }}=t_{R T S}+t_{C T S}+3 t_{\text {SIFS }}+t_{\text {acc }}
$$

Where $\mathrm{t}_{\mathrm{RTS}}$ and ${ }^{t}$ CTS are the time consumed on RTS and CTS, respectively and $t_{S I F S}$ is the SIFS period. $t_{a c c}$ is the time taken due to access contention.

The channel occupation is mainly dependent upon the medium access contention, and the number of packet collisions. That is, $C_{o c c}$ is strongly related to the congestion around a given node.

$C_{o c c}$ can become relatively large if congestion is incurred and not controlled, and it can dramatically decrease the capacity of a congested link.

\subsection{Total Congestion Metric}

The Total Congestion Metric (TCM) can be estimated from the obtained queue length and the channel contention.

$$
T C M=P R\left(Q_{1}\right)+C_{o c c}
$$




\subsection{Agent Based Congestion Control Routing}

The agent based congestion routing Architecture can be explained from the following figure:

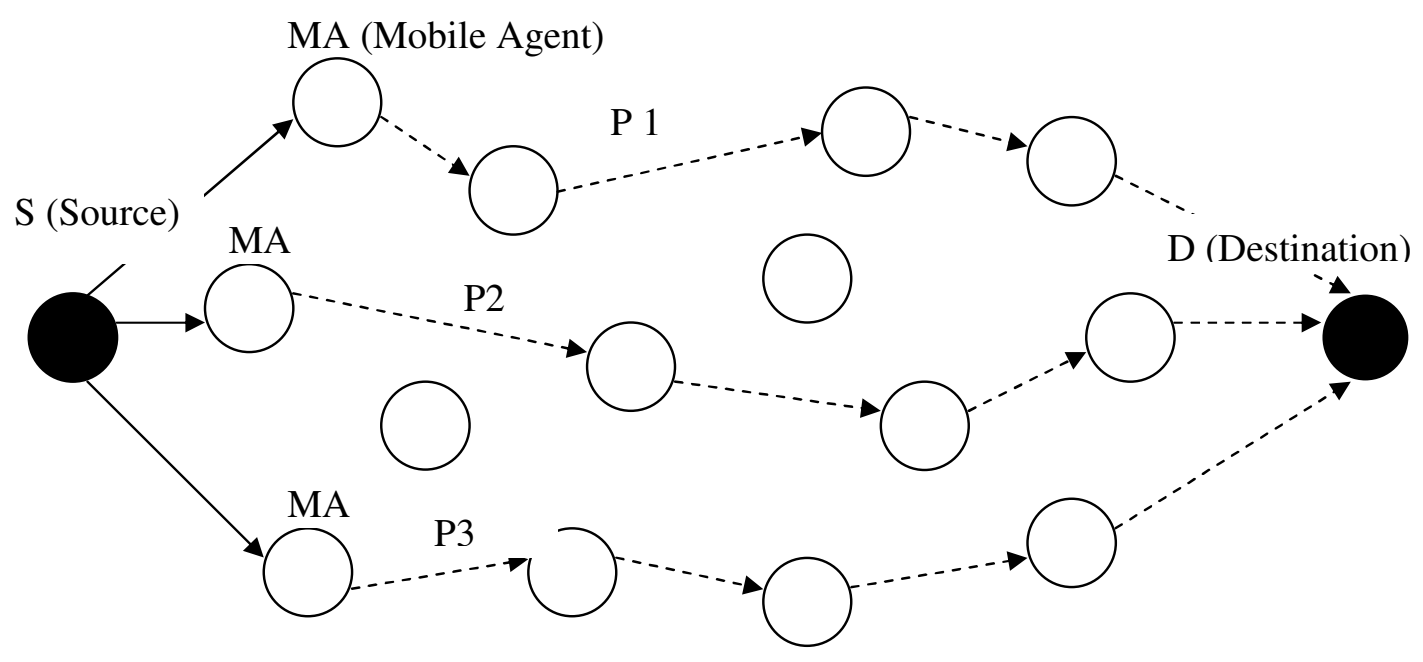

Figure 1 Agent Based Congestion Routing Architecture

Step 1: The source $\mathrm{S}$ checks the number of available one hop neighbors and clones the Mobile Agent (MA) to that neighbors.

Step 2: The Mobile Agent selects the shortest path of the route to move towards the destination $\mathrm{D}$ as given in the figure 1 such as $\mathrm{P} 1, \mathrm{P} 2$ and $\mathrm{P} 3$.

Step 3: The MA1 moves towards the destination D in a hop-by-hop manner in the path P1 and MA2 in P2 and MA3 in P3 respectively.

Step 4: Then the MA1 calculates the TCM1 of that path P1 and similarly MA2 calculates the TCM2 of P2 and MA3 calculates the TCM3 of P3.

Step 5: Now the destination D sends the total congestion metrics TCM1, TCM2 and TCM3 of the paths $\mathrm{P} 1, \mathrm{P} 2$ and $\mathrm{P} 3$ respectively to the source.

Step 6: Now the source selects path using min (TCM1, TCM2, and TCM3) and sends the data through the corresponding path which has the minimum congestion. 
International Journal of Wireless \& Mobile Networks (IJWMN) Vol. 4, No. 2, April 2012

\section{Simulation Results}

\subsection{Simulation Model and Parameters}

We use NS2 [18] to simulate our proposed technique. In the simulation, the channel capacity of mobile hosts is set to the same value: $11 \mathrm{Mbps}$. In the simulation, mobile nodes move in a 1000 meter x 1000 meter region for 50 seconds simulation time. Initial locations and movements of the nodes are obtained using the random waypoint (RWP) model of NS2. It is assumed that each node moves independently with the same average speed. All nodes have the same transmission range of 250 meters. The node speed is $5 \mathrm{~m} / \mathrm{s}$. and pause time is 5 seconds. In the simulation, for class 1 traffic video is used and for class 2 and Class3, CBR and FTP are used respectively.

The simulation settings and parameters are summarized in table 2.

Table 2. Simulation Settings

\begin{tabular}{|l|l|}
\hline No. of Nodes & $10,20,50$ and 100 \\
\hline Area Size & 1000 X 1000 \\
\hline Mac & $802.11 \mathrm{e}$ \\
\hline Radio Range & $250 \mathrm{~m}$ \\
\hline Simulation Time & $50 \mathrm{sec}$ \\
\hline Routing Protocol & AODV \\
\hline Traffic Source & CBR and Video \\
\hline Video Trace & JurassikH263-256k \\
\hline Packet Size & 512 byte \\
\hline Mobility Model & Random Way Point \\
\hline Speed & $5 \mathrm{~m} / \mathrm{s}$ \\
\hline Pause time & 5 sec \\
\hline MSDU & 2132 \\
\hline Varying Rates & $250 \mathrm{~kb}, 500 \mathrm{~kb}, \ldots . . .1000 \mathrm{~Kb}$ \\
\hline Varying No. of Flows & $2,4,6,8$ and 10 \\
\hline
\end{tabular}

\subsection{Performance Metrics}

The performance of proposed Agent Based Congestion Control (ABCC) technique with the Hop by Hop algorithm [9], Cluster Based Congestion Control (CBCC) [11], Congestion-Aware Routing Protocol for Mobile Ad Hoc Networks (CARM) [2], Ad-hoc On-Demand Distance Vector (AODV) Routing [33], Congestion Aware Routing Protocol (CARP) [1], QoS Architecture for Resource Provisioning and Rate Control (QARP-RC) [34] has been compared. The performance is evaluated mainly, according to the following metrics.

Packet Delivery Fraction: It is the ratio of the number of packets received successfully and the total number of packets sent.

Throughput: It is the number of packets received successfully. 
International Journal of Wireless \& Mobile Networks (IJWMN) Vol. 4, No. 2, April 2012

Average end-to-end delay: The end-to-end-delay is averaged over all surviving data packets from the sources to the destinations.

\subsection{Results}

\section{A. Effect of Varying Rates}

In the initial experiment, we measure the performance of the proposed technique by varying the rate as $250,500,750$ and $1000 \mathrm{~Kb}$.

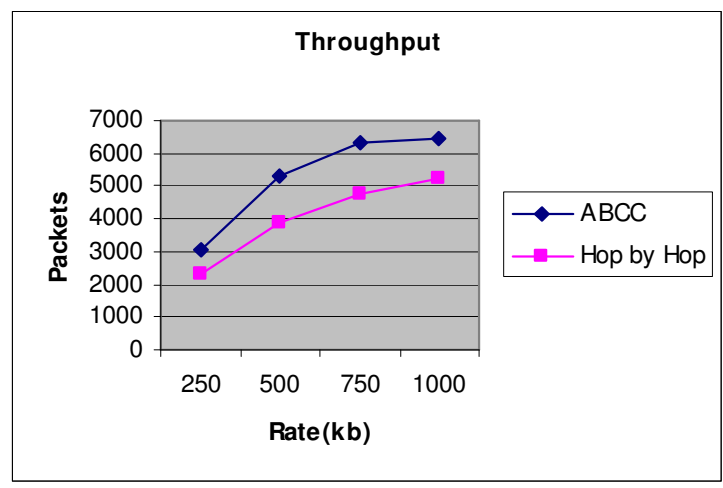

Fig. 2 Rate Vs Throughput for 10 nodes

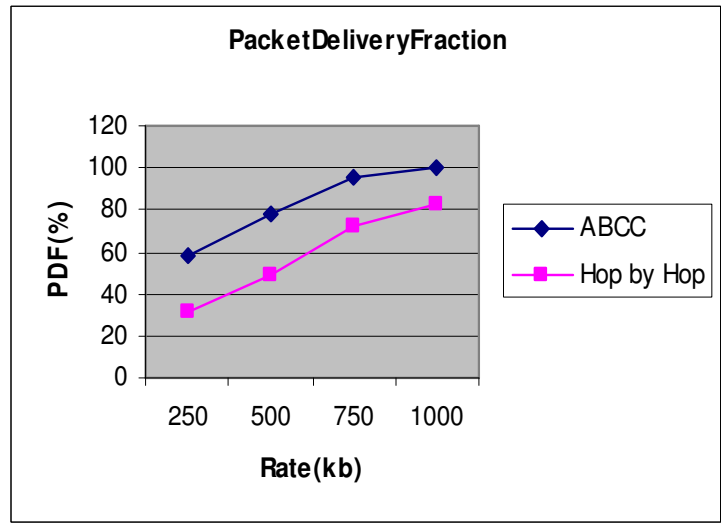

Fig. 3 Rate Vs Packet Delivery Fraction for 20 nodes

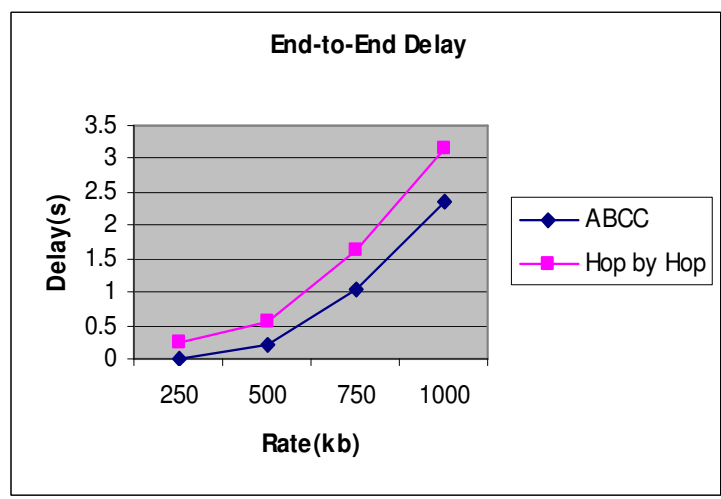

Fig. 4 Rate Vs End-to-End Delay for 50 nodes 
International Journal of Wireless \& Mobile Networks (IJWMN) Vol. 4, No. 2, April 2012

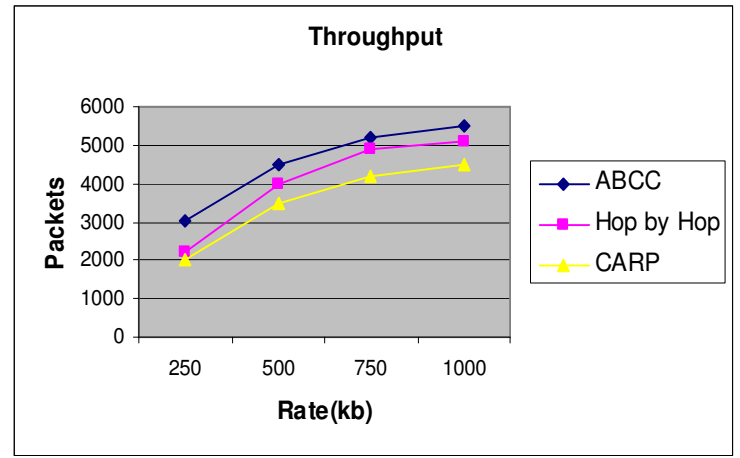

Figure 5 Rate Vs Throughput for 100 nodes

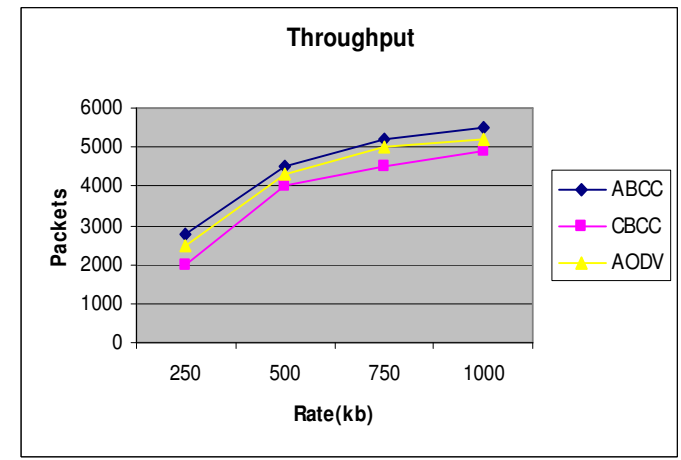

Figure 6 Rate Vs Throughput for 100 nodes

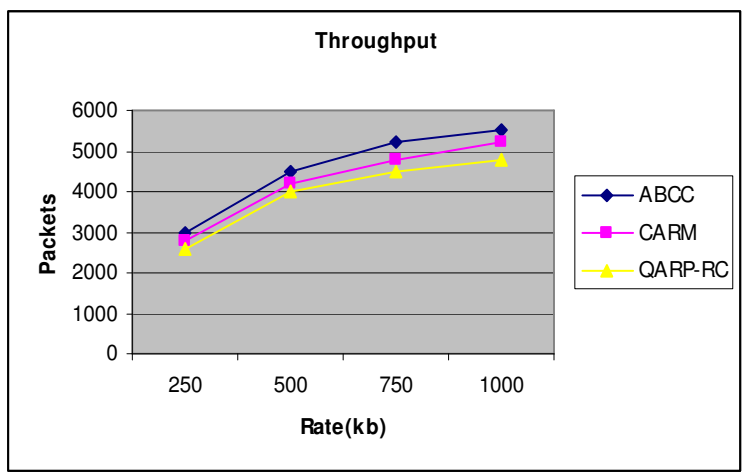

Figure 7 Rate Vs Throughput for 100 nodes

Fig 2, 5, 6, 7 gives the throughput of the proposed technique when the rate is increased. As we can see from the figure, the throughput is more in the case of $\mathrm{ABCC}$ when compared to the existing technique.

From Fig 3, we can see that the packet delivery fraction for ABCC is more, when compared to the Hop by Hop algorithm.

From Fig 4, we can see that the average end-to-end delay of the proposed $\mathrm{ABCC}$ technique is less when compared to the Hop by Hop algorithm. 
International Journal of Wireless \& Mobile Networks (IJWMN) Vol. 4, No. 2, April 2012

\section{B. Effect of varying Flows}

In the next experiment, we compare our proposed technique by varying the number of flows as 2, 4, 6, 8 and 10 .

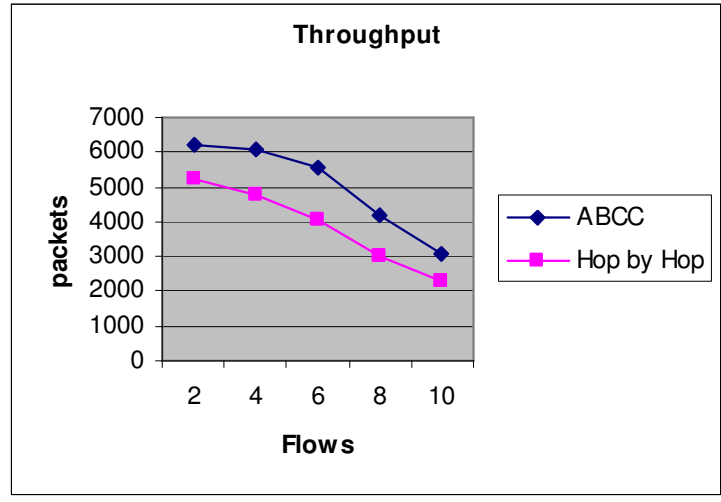

Fig. 8 Flows Vs Throughput for 10 nodes

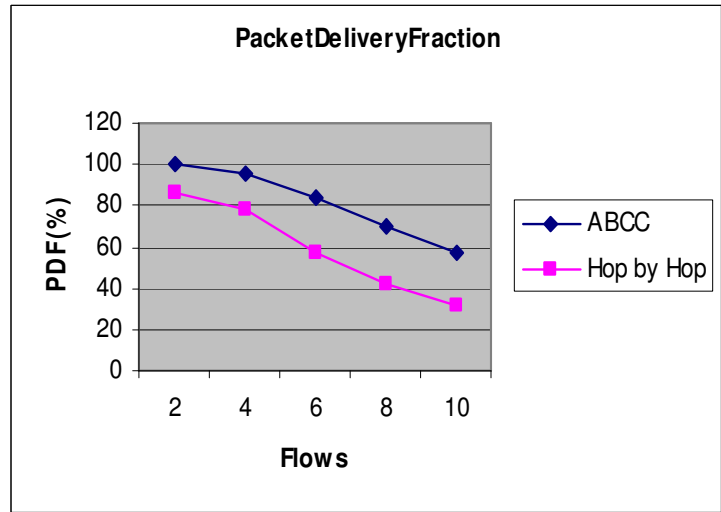

Fig. 9 Flows Vs Packet Delivery Fraction for 20 nodes

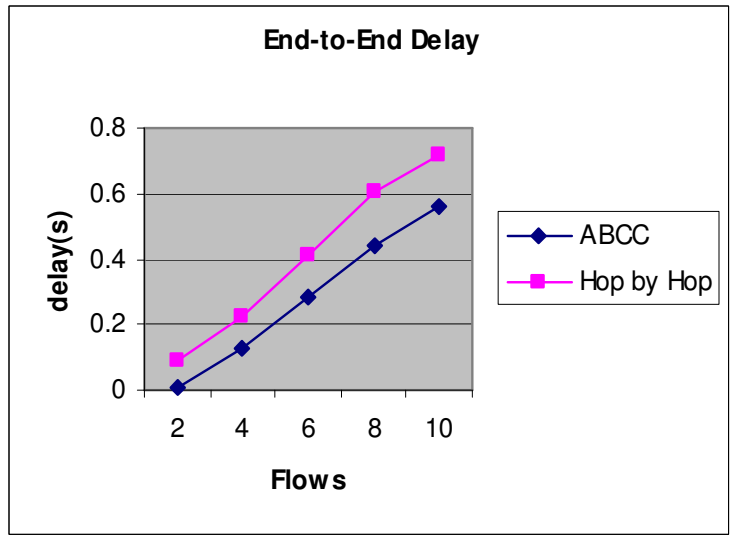

Fig. 10 Flows Vs End-to-End Delay for 50 nodes

\section{0 nodes}


International Journal of Wireless \& Mobile Networks (IJWMN) Vol. 4, No. 2, April 2012

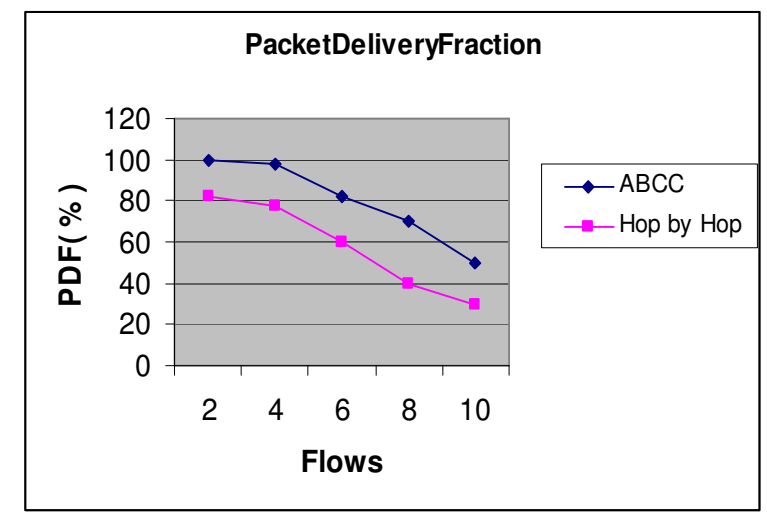

Figure 11 Flows Vs Packet Delivery Fraction for 100 nodes

From Figure 11, it is seen that the packet delivery fraction for ABCC is more, when compared to the Hop by Hop algorithm.

\section{0 nodes}

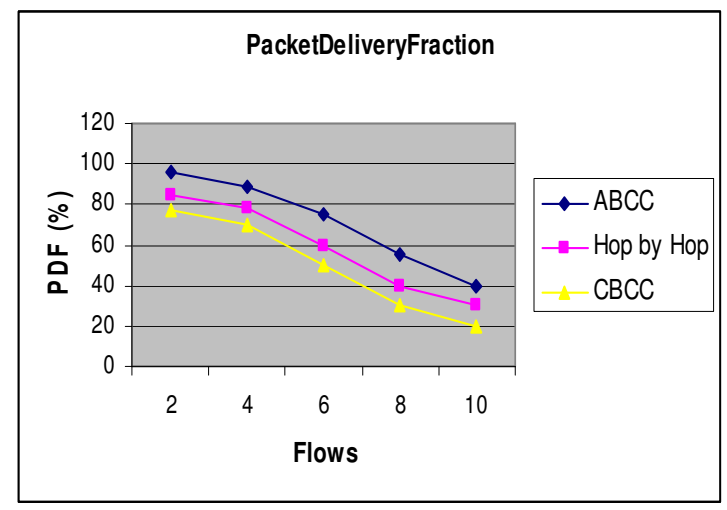

Figure 12 Flows Vs Packet Delivery Fraction for 100 nodes

From Figure 12, it is seen that the packet delivery fraction for $\mathrm{ABCC}$ is more, when compared to the Hop by Hop algorithm and CBCC.

\section{0 nodes}

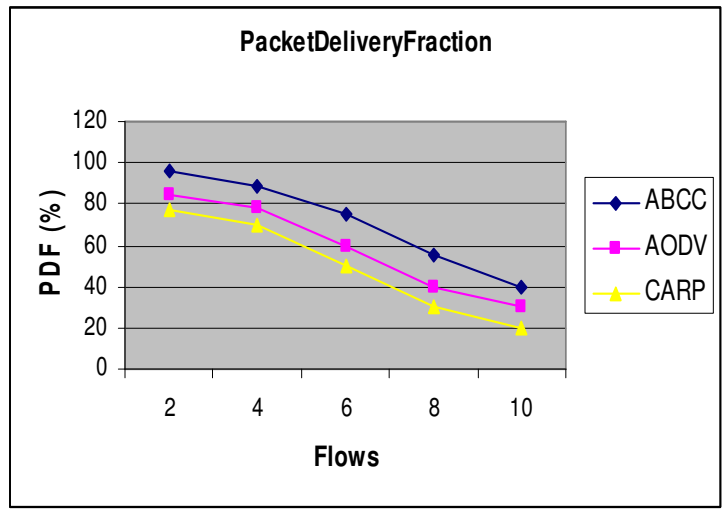

Figure 13 Flows Vs Packet Delivery Fraction for 100 nodes 
International Journal of Wireless \& Mobile Networks (IJWMN) Vol. 4, No. 2, April 2012

Fig 8 gives the throughput of the proposed technique when the flow is increased. As we can see from the figure, the throughput is more in the case of $\mathrm{ABCC}$ when compared to the Hop by Hop algorithm.

From Fig 9, we can see that the packet delivery fraction for ABCC is more, when compared to the Hop by Hop algorithm.

From Fig 10, we can see that the average end-to-end delay of the proposed ABCC technique is less when compared to the Hop by Hop algorithm.

From Figure 13, it is seen that the packet delivery fraction for $\mathrm{ABCC}$ is more, when compared to the AODV algorithm and CARP algorithm.

\section{Conclusion}

In this paper, we have developed of an agent based congestion control technique. In our technique, the information about network congestion is collected and distributed by mobile agents (MA). A mobile agent starts from every node and moves to an adjacent node at every time. A node visited next is selected at the equivalent probability. The MA brings its own history of movement and updates the routing table of the node it is visiting. The MA updates the routing table of the node it is visiting. In this technique, the node is classified in one of the four categories depending on whether the traffic belongs to background, best effort, video or voice AC respectively. Then MA estimates the queue length of the various traffic classes and the channel contention of each path. Then this total congestion metric is applied to the routing protocol to select the minimum congested route in the network., a mobile agent based congestion control AODV routing protocol reduces the end-to-end delay and the number of route discovery requests, balances the traffic load. By simulation results, we have shown that our proposed technique attains high delivery ratio and throughput with reduced delay when compared with the existing technique.

\section{Reference}

[1] Baboo, S.S., Narasimhan, B.: A Hop-by-Hop Congestion-Aware Routing Protocol for Heterogeneous Mobile Ad-hoc Networks. International Journal of Computer Science and Information Security. (2009).

[2] Chen, X., Jones, H.M., Jayalath, A.D.S. : Congestion-Aware Routing Protocol for Mobile Ad Hoc Networks. IEEE $66^{\text {th }}$ Conference in Vehicular Technology. (2007).

[3] Lochert, C., Scheuermann, B., Mauve, M.: A Survey on Congestion Control for Mobile Ad-Hoc Networks. Wireless Communications and Mobile Computing, InterScience. (2007)

[4] http://en.wikipedia.org/wiki/Congestion_control

[5] http://www.linktionary.com/c/congestion.html

[6] Tran, D.A., Raghavendra, H.: Congestion Adaptive Routing in Mobile Ad Hoc Networks. IEEE Transactions on Parallel and Distributed Systems. (2006)

[7] Lien, Y.N., Hsiao, H.C.: A New TCP Congestion Control Mechanism over Wireless Ad Hoc Networks by Router-Assisted Approach. 27th IEEE International Conference on Distributed Computing Systems Workshops. (2007)

[8] Sun, W., Wen, T., Guo, Q.: A Performance Comparison of Equation-Based and GAIMD Congestion Control in Mobile Ad Hoc Networks. International Conference on Computer Science and Software Engineering. (2008) 
International Journal of Wireless \& Mobile Networks (IJWMN) Vol. 4, No. 2, April 2012

[9] Yi, Y., Shakkottai, S.: Hop-by-Hop Congestion Control Over a Wireless Multi-Hop Network. IEEE/ACM Transactions on Networking. (2007)

[10] Akyol, U., Andrews, M., Gupta, P., Hobby, J., Saniee, I., Stolyar, A.: Joint Scheduling and Congestion Control in Mobile Ad-Hoc Networks. Proceedings of IEEE INFOCOM. (2008)

[11] Karunakaran, S., Thangaraj, P.: A Cluster Based Congestion Control Protocol for Mobile Adhoc Networks. International Journal of Information Technology and Knowledge Management. Vol. 2, No. 2, pp. 471-474.(2010).

[12] Malika, B., Mustapha, L., Abdelaziz, M., Nordine, T., Mehammed, D., Rachida, A.: Intelligent Routing and Flow Control In MANETs. Journal of Computing and Information Technology. doi: 10.2498/cit.1001470

[13] Li, J., Li, Z., Mohapatra, P.: APHD: End-to-End Delay Assurance in 802.11e Based MANETs. Mobile and Ubiquitous Systems - Workshops, 3rd Annual International Conference. pp. 1-8. (2006)

[14] Lee, J.F., Liao, W., Chen, M.C. : A Differentiated Service Model for Enhanced Distributed Channel Access (EDCA) of IEEE 802.11e WLANs. in Proc. IEEE Globecom. (2005)

[15] Ksentini, A., Naimi, M., Gueroui, A.: Toward an Improvement of H.264 Video Transmission over IEEE 802.11e through a Cross-Layer Architecture. IEEE Communications Magazine. (2006)

[16] Wu, Y-J., Chiu, J-H., Sheu, T-L. : A Modified EDCA with Dynamic Contention Control for RealTime Traffic in Multi-hop Ad Hoc Networks. Journal of Information Science and Engineering. vol. 24, pp.1065-1079. (2008).

[17] Flaithearta, P.O, Melvin, H.:802.11e EDCA Parameter Optimization Based on Synchronized Time. MESAQIN. (2009)

[18] Network Simulator, http://www.isi.edu/nsnam/ns

[19] Venkatasubramanian, S., Gopalan, N.P.: A Quality of service architecture for resource provisioning and rate control in mobile ad hoc networks. International Journal of Ad hoc, Sensor \& Ubiquitous Computing (IJASUC). Vol.1, No.3.(2010).

[20] Sulthani, R.M., Rao, D.S.: Design of an Efficient QoS Architecture (DEQA) for Mobile Ad hoc Networks. ICGST-CNIR Journal. Vol. 8, Issue 2. (2009).

[21] Chen, L., Lowy, S.H., Chiangz, M., Doyley, J.C.: Cross-layer Congestion Control, Routing and Scheduling Design in Ad Hoc Wireless Networks. Proc., IEEE, 25 ${ }^{\text {th }}$ international conference on computer communication, INFOCOM. pp 1 - 13. (2007)

[22] Wang, X., Perkins, D.: Cross-layer Hop-by-hop Congestion Control in Mobile Ad Hoc Networks. Proc., IEEE, wireless communication and networking conference, WCNC. pp 2456 - 2461. (2008)

[23] Nishimura, K., Takahashi, K.: A Multi-Agent Routing Protocol with Congestion Control for MANET. Proceedings 21st European Conference on Modeling and Simulation (ECMS). (2007)

[24] Bhadauria SS,Sharma V.,Framework and Implimentation of an Agent Based Congestion Control Technique for Mobile Ad-hoc Network ,ICAC3 2011, CCIS 125, pp. 318-327, 2011.

[25] Sharma V, Bhadauria SS," Agent Based Congestion Control Routing for Mobile Ad-hoc Network”Wimon-2011,CCIS,Volume 197, pp.324-333.

[26] Hong Li, Chu Dan, Wang Min, Li Shurong,"'Mobile Agent Based Congestion Control AODV Routing Protocol" Wireless Communications, Networking and Mobile Computing, 2008. WiCOM '08. 4th International Conference, Dalian,12-14 Oct. 2008.

[27] RAMANATHAN R, REDI J. "A Brief Overview of Ad Hoc Networks Challenges and Directions" IEEE Communications Magazine 2002, 40(5),20-22.

[28] HASSANEIN H, ZHOU A. Routing with Load Balancing in Wireless Ad- Hoc Networks[C]. Proceedings of the 4th ACM International Workshop on Modelling, Analysis , and Simulation of Wireless and Mobile. Systems, Rome,2001:89-96. 
International Journal of Wireless \& Mobile Networks (IJWMN) Vol. 4, No. 2, April 2012

[29] Thomas Kunz,” Energy-Efficient MANET Routing: Ideal vs. Realistic Performance”, International conference on wireless communication and mobile computing (IWCMC), pp 786 - 793, 2008.

[30] Vinay Rishiwal, Shekhar Verma and S. K. Bajpai, "QoS Based Power Aware Routing in MANETs," International Journal of Computer Theory and Engineering (IJCTE), vol. 1, no. 1, pp. 47-54, 2009.

[31] PERKINS C E, ROYER E M, DAD S R. Ad Hoc On-demand Distance Vector(AODV) Routing[C]. IEEE Workshop on Mobile Computing Systems and Applications, 1999:90-100.

[32] Ali El-Haj-Mahmoud, Rima Khalaf, Ayman Kayssi, "PERFORMANCE COMPARISON OF THE AODV AND DSDV ROUTING PROTOCOLS IN MOBILE AD HOC NETWORKS" Communication Systems and Networks, September 9 - 12,Spain, 2002

[33] C. E. Perkins and E. M. Royer, "The Ad Hoc On-Demand Distance-Vector Protocol (AODV)," In Ad Hoc Networking, C. E. Perkins (Ed.), Addison-Wesley, pp. 173-219, 2001.

[34] Venkatasubramanian, S., Gopalan, "N.P.: A Quality of service architecture for resource provisioning and rate control in mobile ad hoc networks", International Journal of Ad hoc, Sensor \& Ubiquitous Computing (IJASUC),Vol. 1, No.3, pp. 106-120, 2010.

Short Biography

Vishnu Sharma working as a faculty in CSE Deptt. of Jaypee University of Engg and Technology and Presently, he is a Ph.D Candidate at M.P.Technical University,He has about 13 years of teaching experience. He has published more than fifteen papers in the area of Mobile Ad-hoc Networks \& Mobile Computing at National/International Level.He has Published the books on Mobile Computing and Advanced Mobile Computing in UPTU,Lucknow(UP) and IP University,New Delhi. He is a life time member of International Association of Computer Science and Information Technology (IACSIT), Singapore and member of CSI. His area of

interest includes Mobile Computing,Computer Networks and Communication Technologies,Cryptography and Network Security.

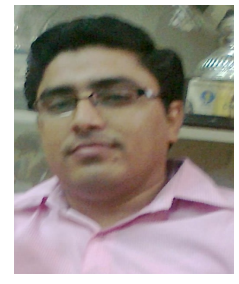

Dr.Sarita Singh Bhadauria She is a Professor in Department of Electronics MITS,Gwalior (M.P.) India , She has about 25 years of teaching and research experience.She has Published more than fifty papers in the area of Digtal Image Processing, Computer Networks,Mobile Ad-hoc Networks, Mobile Communication and Cryptography and Network Security and Digital Communication at National/International Level.She is a life time member of ISTE, IETE, IEEE, CSI, HAM RADIO. Her areas of interest includes Wireless Communication and Digtal

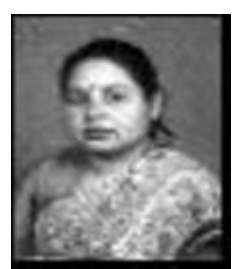

Image Processing, Computer Networks,and Mobile Ad-hoc Networks, Mobile Communication and Cryptography and Network Security and Digital Communication and Communication Technologies. 